PROCEEDINGS OF THE

AMERICAN MATHEMATICAL SOCIETY

Volume 128, Number 5, Pages 1405-1408

S 0002-9939(99)05268-5

Article electronically published on August 5, 1999

\title{
TOEPLITZ ALGEBRAS ON DISCRETE ABELIAN QUASILY ORDERED GROUPS
}

\author{
XU QINGXIANG
}

(Communicated by David R. Larson)

\begin{abstract}
In this note, Toeplitz operators on discrete abelian quasily ordered groups are studied, and a theorem of R. Douglas is generalized.
\end{abstract}

\section{INTRODUCTION}

Throughout the note, we assume that $G$ is a discrete abelian group. For any subset $G_{+}$of $G$, we say that $\left(G, G_{+}\right)$is a quasi-partial ordered group if $0 \in G_{+}, G_{+}+$ $G_{+} \subseteq G_{+}$and $G=G_{+}-G_{+}$; further, $\left(G, G_{+}\right)$is referred to as a quasily ordered group if $G=G_{+} \cup\left(-G_{+}\right)$. Note when $G_{+}^{0}=G_{+} \cap\left(-G_{+}\right)=\{0\}$, then a quasipartial ordered group ( resp. quasily ordered group ) $\left(G, G_{+}\right)$is known as a partially ordered ( resp. ordered) group.

Let $\left\{e_{g} \mid g \in G\right\}$ be the usual orthonormal basis for $\ell^{2}(G)$, where

$$
e_{g}(h)=\left\{\begin{array}{ll}
1, & \text { if } g=h, \\
0, & \text { otherwise, }
\end{array} \quad \text { for } g, h \in G .\right.
$$

For any $E \subseteq G$, let $\ell^{2}(E)$ be the closed subspace of $\ell^{2}(G)$ generated by $\left\{e_{g} \mid g \in E\right\}$; its projection is denoted by $p^{E}$. For any $g \in G$, we define a unitary $u_{g}$ on $\ell^{2}(G)$ by $u_{g}\left(e_{h}\right)=e_{g+h}$ for $h \in G$. For any subset $E$ of $G$, the $C^{*}$-algebra generated by $\left\{p^{E} u_{g} p^{E} \mid g \in G\right\}$ is denoted by $\mathcal{T}^{E}(G)$ and is called the Toeplitz algebra with respect to $E$.

Toeplitz algebras on the quarter-planes have been studied by many mathematicians; see [1] and 2] for example. Associated with such Toeplitz algebras are the usual quasily ordered groups $\left(\mathbb{Z}^{2}, \mathbb{Z}_{\alpha}^{2}\right)$ for $\alpha \in \mathbb{R}^{1}$, where $\mathbb{Z}_{\alpha}^{2}=\{(m, n) \in$ $\left.\mathbb{Z}^{2} \mid \alpha m+n \geq 0\right\}$. R. Douglas proved in [2], among other things, that when $\alpha$ is a rational number $\mathcal{T}^{\mathbb{Z}_{\alpha}^{2}}\left(\mathbb{Z}^{2}\right) \cong \mathcal{T}^{\mathbb{Z}_{+}}(\mathbb{Z}) \otimes C(T)$, where $T$ is the unit circle in $\mathbb{C}^{1}$ and $\mathcal{T}^{\mathbb{Z}_{+}}(\mathbb{Z})$ is the classical Toeplitz algebra (see Corollary 4 below). In this note, under a setting of quasi-partial ordered groups, we generalize such a result.

Received by the editors February 20, 1998 and, in revised form, June 30, 1998

1991 Mathematics Subject Classification. Primary 47B35.

Key words and phrases. Discrete abelian group, quasily ordered group, Toeplitz operator.

This project was partially supported by the National Natural Science Foundation of China (No. 19901019) and the Youth Science Foundation of Shanghai Higher Education (No. 98QN75). 


\section{The MAIN RESUlts}

Let $G$ be a discrete abelian group and $\left(G, G_{+}\right)$a quasily ordered group. By an isometric representation $(V, H)$ of $G_{+}$, we mean $H$ is a Hilbert space and $V: G_{+} \rightarrow$ $\mathbb{B}(H)$ is a map satisfying

(1) $V(0)=1, V^{*}(x) V(x)=1$ for $x \in G_{+}$;

(2) $V(x+y)=V(x) V(y)$ for $x, y \in G_{+}$;

(3) $V(x) V(x)^{*}=1$ for $x \in G_{+}^{0}$.

Lemma 1 (cf. 9], Theorem 3.5). Let $G$ be a discrete abelian group and $\left(G, G_{+}\right)$a quasily ordered group. Then for any isometric representation $V: G_{+} \rightarrow \mathbb{B}(H)$, there is a $C^{*}$-algebra morphism $\pi_{V}: \mathcal{T}^{G_{+}}(G) \rightarrow \mathbb{B}(H)$ such that $\pi_{V}\left(p^{G_{+}} u_{g} p^{G_{+}}\right)=$ $V(g)$ for all $g \in G_{+}$.

Remark. (1) Let $\left(G, G_{+}\right)$and $\left(E, E_{+}\right)$be two quasily ordered groups. If there is an isomorphism of groups $\rho: G \rightarrow E$ such that $\rho\left(G_{+}\right)=E_{+}$and $\rho\left(G_{+}^{0}\right)=E_{+}^{0}$, then by the above Lemma, we know that $\mathcal{T}^{G_{+}}(G) \cong \mathcal{T}^{E_{+}}(E)$.

(2) Let $\widehat{G}$ be the dual group of $G$. Since $G$ is discrete and abelian, $\widehat{G}$ is compact and it is connected if and only if $G$ is torsion-free. By [6], Proposition 7.1.6, we know that the reduced group $C^{*}$-algebra $C_{r}^{*}(G)=\mathcal{T}^{G}(G) \cong_{\rho} C(\widehat{G})$, with the property $\rho\left(u_{g}\right)=\varepsilon_{g}$ for any $g \in G$, where $\varepsilon_{g}(\gamma)=\gamma(g)$ for $\gamma \in \widehat{G}$. By the Stone-Weierstrass Theorem, $C(\widehat{G})$ is generated by $\left\{\varepsilon_{g} \mid g \in G\right\}$. Applying the above Lemma to $(G, G)$, we get the following Corollary.

Corollary 2 (cf. 3], Lemma 1.2). Let $G$ be a discrete abelian group. Then for any unitary representation $(V, H)$ of $G$, there is a $C^{*}$-algebra morphism $\pi_{V}: C(\widehat{G}) \rightarrow$ $\mathbb{B}(H)$ such that $\pi_{V}\left(\varepsilon_{g}\right)=V(g)$ for all $g \in G$.

Now let $\left(G, G_{+}\right)$be a quasily ordered group. By definition, we know that $G=G_{+} \cup\left(-G_{+}\right)$and $0 \in G_{+}^{0}=G_{+} \cap\left(-G_{+}\right)$. Let $G_{+}^{*}=G_{+} \backslash G_{+}^{0}$; then it is easy to show that

$$
G_{+}^{*}+G_{+}=G_{+}^{*}, \quad G=G_{+}^{*} \cup G_{+}^{0} \cup\left(-G_{+}^{*}\right) .
$$

So if we set $G_{1}=G_{+}^{*} \cup\{0\}$, then for any $x \in G_{+}^{0}$ and $y \in G_{+}^{*}$, we have $x=$ $(x+y)-y \in G_{1}-G_{1}$; therefore $\left(G, G_{1}\right)$ is actually a partially ordered group. Further, by $(*)$ we know that $\left(G / G_{+}^{0}, G_{1} / G_{+}^{0}\right)$ is an ordered group.

Next we generalize R. Douglas's result to a more general setting, but first we need a definition.

Definition. Let $G$ be a discrete abelian group and $\left(G, G_{+}\right)$a quasily ordered group. A morphism of groups $\phi: G \rightarrow G_{+}^{0}$ is said to be a deformation retraction if $\phi(h)=h$ for all $h \in G_{+}^{0}$.

Theorem 3. Let $G$ be a discrete abelian group and $\left(G, G_{+}\right)$a quasily ordered group. Denote $G / G_{G_{+}^{0}}=[G]$ and $G_{1} /_{G_{+}^{0}}=\left[G_{1}\right]$. If there is a deformation retraction $\phi: G \rightarrow G_{+}^{0}$, then $\mathcal{T}^{G_{+}}(G) \cong \mathcal{T}^{\left[G_{1}\right]}([G]) \otimes C\left(\widehat{G_{+}^{0}}\right)$.

Proof. For any $g \in G$, let $T_{g}^{G_{+}}=p^{G_{+}} u_{g} p^{G_{+}}, T_{[g]}^{\left[G_{1}\right]}=p^{\left[G_{1}\right]} u_{[g]} p^{\left[G_{1}\right]}$. For $g \in G_{+}$, define

$$
V(g)=T_{[g-\phi(g)]}^{\left[G_{1}\right]} \otimes \varepsilon_{\phi(g)}=T_{[g]}^{\left[G_{1}\right]} \otimes \varepsilon_{\phi(g)}
$$


then $V$ is an isometric representation of $G_{+}$. By Lemma 1, we know that there is a $C^{*}$-algebra morphism $\pi: \mathcal{T}^{G_{+}}(G) \rightarrow \mathcal{T}^{\left[G_{1}\right]}([G]) \otimes C\left(\widehat{G_{+}^{0}}\right)$ such that

$$
\pi\left(T_{g}^{G_{+}}\right)=V(g)=T_{[g]}^{\left[G_{1}\right]} \otimes \varepsilon_{\phi(g)} \text { for any } g \in G_{+},
$$

which implies

$$
\pi\left(T_{g}^{G_{+}}\right)=T_{[g]}^{\left[G_{1}\right]} \otimes \varepsilon_{\phi(g)} \text { for any } g \in G .
$$

On the other hand, by Lemma 1 and Corollary 2, we know that there are $C^{*}$ algebra morphisms $\rho: \mathcal{T}^{\left[G_{1}\right]}([G]) \rightarrow \mathcal{T}^{G_{+}}(G)$ and $\lambda: C\left(\widehat{G_{+}^{0}}\right) \rightarrow \mathcal{T}^{G_{+}}(G)$, such that

$$
\rho\left(T_{[g]}^{\left[G_{1}\right]}\right)=T_{g-\phi(g)}^{G_{+}} \text {for any } g \in G, \quad \lambda\left(\varepsilon_{h}\right)=T_{h}^{G_{+}} \text {for any } h \in G_{+}^{0} .
$$

It is easy to show that for any $g \in G_{1}$ and $h \in G_{+}^{0}, \rho\left(T_{[g]}^{\left[G_{1}\right]}\right) \lambda\left(\varepsilon_{h}\right)=\lambda\left(\varepsilon_{h}\right) \rho\left(T_{[g]}^{\left[G_{1}\right]}\right)$, and since $C\left(\widehat{G_{+}^{0}}\right)$ is nuclear, by [5], Corollary T.6.9, we know that there is a $C^{*}$ algebra morphism $\rho \otimes \lambda: \mathcal{T}^{\left[G_{1}\right]}([G]) \otimes C\left(\widehat{G_{+}^{0}}\right) \rightarrow \mathcal{T}^{G_{+}}(G)$ such that

$$
(\rho \otimes \lambda)\left(T_{[g]}^{\left[G_{1}\right]} \otimes \varepsilon_{h}\right)=T_{g-\phi(g)}^{G_{+}} T_{h}^{G_{+}} \text {for any } g \in G \text { and } h \in G_{+}^{0} .
$$

For any $g \in G_{+}$and $h \in G_{+}^{0}$,

$$
((\rho \otimes \lambda) \circ \pi)\left(T_{g}^{G_{+}}\right)=(\rho \otimes \lambda)\left(T_{[g]}^{\left[G_{1}\right]} \otimes \varepsilon_{\phi(g)}\right)=T_{g-\phi(g)}^{G_{+}} T_{\phi(g)}^{G_{+}}=T_{g}^{G_{+}},
$$

and

$$
\begin{aligned}
& (\pi \circ(\rho \otimes \lambda))\left(T_{[g]}^{\left[G_{1}\right]} \otimes \varepsilon_{h}\right)=\pi\left(T_{g-\phi(g)}^{G_{+}} T_{h}^{G_{+}}\right) \\
& =\pi\left(T_{g-\phi(g)}^{G_{+}}\right) \pi\left(T_{h}^{G_{+}}\right)=\left(T_{[g]}^{\left[G_{1}\right]} \otimes 1\right)\left(1 \otimes \varepsilon_{h}\right)=T_{[g]}^{\left[G_{1}\right]} \otimes \varepsilon_{h} .
\end{aligned}
$$

Therefore, $\mathcal{T}^{G_{+}}(G) \cong \mathcal{T}^{\left[G_{1}\right]}([G]) \otimes C\left(\widehat{G_{+}^{0}}\right)$.

Corollary 4 ([10]). Let $p, q \in \mathbb{Z}$ such that there exist $r, s \in \mathbb{Z}$ with the property $r p+s q=1$. Denote $G=\mathbb{Z}^{2}$ and $G_{+}=\mathbb{Z}_{p, q}^{2}=\left\{(m, n) \in \mathbb{Z}^{2} \mid p m+q n \geq 0\right\}$. Then $\mathcal{T}^{\mathbb{Z}_{p, q}^{2}}\left(\mathbb{Z}^{2}\right) \cong \mathcal{T}^{\mathbb{Z}_{+}}(\mathbb{Z}) \otimes C(T)$.

Proof. Define a deformation retraction $\phi: G \rightarrow G_{+}^{0}$ by

$$
\phi(m, n)=(-q(-s m+r n), p(-s m+r n)) .
$$

Since $r p+s q=1$, we know that $\left\{-s m+r n \mid(m, n) \in \mathbb{Z}^{2}\right\}=\mathbb{Z}$. Further, if $(k, l) \in G_{+}^{0}$, i.e. $p k+q l=0$, then $k=q a$ and $l=-p a$ for some $a \in \mathbb{Z}$. Choose $m, n \in \mathbb{Z}$ such that $s m-r n=a$, then $\phi(m, n)=(k, l)$. Therefore,

$$
G_{+}^{0}=\left\{(-q(-s m+r n), p(-s m+r n)) \mid(m, n) \in \mathbb{Z}^{2}\right\} .
$$

So if we set $\rho: G_{+}^{0} \rightarrow \mathbb{Z}$ by $\rho(-q(-s m+r n), p(-s m+r n))=-s m+r n$, then $\rho$ is an isomorphism of groups. By Corollary 2, we know $C\left(\widehat{G_{+}^{0}}\right) \cong C(T)$. Obviously, $G_{1}=\{(m, n) \mid p m+q n>0\} \cup\{(0,0)\}$. Let $\theta:[G] \rightarrow \mathbb{Z}, \theta[(m, n)]=p m+q n$ for $(m, n) \in \mathbb{Z}^{2}$; then $\theta$ is an order isomorphism of groups. By Lemma 1 , we know that $\mathcal{T}^{\left[G_{1}\right]}([G]) \cong \mathcal{T}^{\mathbb{Z}_{+}}(\mathbb{Z})$, so the conclusion holds. 
Remark. Let $\widehat{G}$ be the dual group of $G$; then $\left\{\varepsilon_{x} \mid x \in G\right\}$ is an orthonormal basis for $L^{2}(\widehat{G})$. When $\left(G, G_{+}\right)$is a quasi-partial ordered group, we may also regard $\mathcal{T}^{G_{+}}(G)$ as a subalgebra of $B\left(L^{2}(\widehat{G})\right)$ as follows:

Let $H^{G_{+}}(\widehat{G})$ be the closed subspace of $L^{2}(\widehat{G})$ generated by $\left\{\varepsilon_{x} \mid x \in G_{+}\right\}$; its projection is denoted by $p^{G_{+}}(\widehat{G})$. For any $\varphi \in C(\widehat{G})$, define $T_{\varphi}^{G_{+}}$on $H^{G_{+}}(\widehat{G})$ by $T_{\varphi}^{G_{+}}(f)=p^{G_{+}}(\varphi f)$ for $f \in H^{G_{+}}(\widehat{G})$. The $C^{*}$-algebra generated by $\left\{T_{\varphi}^{G_{+}} \mid \varphi \in\right.$ $C(\widehat{G})\}$ is denoted by $\mathcal{T}_{r}^{G_{+}}(\widehat{G})$ and is also called the Toeplitz algebra with respect to $G_{+}$. Clearly, $\mathcal{T}_{r}^{G_{+}}(\widehat{G})$ is generated by $\left\{T_{\varepsilon_{x}}^{G_{+}} \mid x \in G\right\}$ and it can be regarded as a $C^{*}$-subalgebra of $\mathbb{B}\left(L^{2}(\widehat{G})\right)$ through zero-extension. So if we define a unitary $u: \ell^{2}(G) \rightarrow L^{2}(\widehat{G})$ by $u e_{g}=\varepsilon_{g}$ for $g \in G$, then $u^{*} \circ T_{\varepsilon_{g}}^{G_{+}} \circ u=p^{G_{+}} u_{g} p^{G_{+}}$; therefore the two Toeplitz algebras are unitarily equivalent.

Now let $(G, E)$ be an ordered group, in this case $G$ is torsion-free, so $\widehat{G}$ is connected. For any $\varphi \in C(\widehat{G})$, if it does not vanish anywhere, then by [7] there exist some $x$ in $G$ and $\psi \in C(\widehat{G})$ such that $\varphi=\varepsilon_{x} e^{\psi}$. As observed in [4], such an $x$ is unique. We denote this group element $\operatorname{ind}_{t}(\varphi)$ and call it the topological index of $\varphi$. This index depends only on the connected component of $C(\widehat{G})^{-1}$ in which $\varphi$ lies. The following proposition is stated in [10], and as we know when $\left(G, G_{+}\right)=\left(\mathbb{Z}^{2}, \mathbb{Z}_{p, q}^{2}\right)$, it reduces to [2], Theorem 5 .

Proposition ([10]). Let $\left(G, G_{+}\right)$be a quasily ordered group ( $G$ need not admit a deformation retraction onto $\left.G_{+}\right)$. If $G$ is torsion-free, then for any $\phi \in C(\widehat{G}), T_{\phi}^{G_{+}}$ is invertible if and only if $\phi$ does not vanish anywhere and $\operatorname{ind}_{t}(\phi) \in G_{+}^{0}$.

\section{ACKNOWLEDGEMENT}

The author would like to thank the referee for helpful suggestions.

\section{REFERENCES}

1. E. Park, Index theory and Toeplitz algebras on certain cones in $Z^{2}$, J. Operator Theory 23 (1990), 125-146. MR 91i:47039

2. R. Douglas, Another look at real-valued index theory, Proceedings of Special Year on Operator Theory, Bloomington (1985-86).

3. G. Murphy, Ordered groups and Toeplitz algebras, J. Operator Theory 18 (1987), 303-326. MR 89f: 46132

4. (1991), 1-6. MR 93k:47039

5. N. Wegge-Olsen, K-theory and $C^{*}$-algebra, Oxford University Press Inc., New York, 1993. MR 95c:46116

6. G. Pedersen, $C^{*}$-algebras and their automorphism groups, Academic Press, London, 1979.

7. E. Van Kampen, On almost periodic function of constant absolute value, J. London Math. Soc. 12 (1937), 3-6.

8. Q. Xu and X. Chen, A note on Toeplitz operators on discrete groups, Proc. Amer. Math. Soc. 126 (1998), no. 12, 3625-3631. CMP 98:17

9. - Toeplitz $C^{*}$-algebras on ordered groups and their ideals of finite elements, Proc. Amer. Math. Soc. 127 (1999), no. 2, 553-561. CMP 98:05

10. X. Chen and Q. Xu, Toeplitz operators on discrete abelian groups, preprint.

Department of Mathematics, Shanghai Normal University, Shanghai 200234, People's Republic of China

E-mail address: mathsci@shtu.edu.cn 